\title{
Reviews on cutting-edge topics in toxicology
}

\author{
Hermann M. Bolt
}

Published online: 27 November 2014

(C) Springer-Verlag Berlin Heidelberg 2014

Since some years, our journal initiated a series of review articles on cutting-edge topics in the field of toxicology. Ten of the most cited and downloaded articles are summarized in Table 1. The focus of the articles published in 2012 is oxidative stress, toxicity of zinc, amphetamines, pyrethroid insecticides, cisplatin and nanomaterials. Moreover, the role of estrogen receptors in human disease and alternative methods for skin sensitization have been covered. A key feature of our review articles is that our authors are not limited by strict word counts. Comprehensive manuscripts that cover an entire research field are encouraged. We hope that this type of review is of particular interest to our readers and we are looking forward to further contributions.

Table 1 Ten most cited review articles published in the Archives of Toxicology in 2012

\begin{tabular}{ll}
\hline Focus of the review & References \\
\hline Plant-derived antioxidants & Gulcin (2012) \\
Zinc and human health & Chasapis et al. (2012) \\
Toxicity of amphetamines & Carvalho et al. (2012) \\
Oxidative stress in apoptosis & Matés et al. (2012) \\
Pyrethroid insecticide neurotoxicity & Soderlund et al. (2012) \\
Estrogen receptors in human disease & Burns et al. (2012) \\
Toxicity of magnetic nanoparticles & Kim et al. (2012) \\
Cisplatin-induced nephrotoxicity & dos Santos et al. (2012) \\
Toxicokinetics of nanomaterials & Landsiedel et al. (2012) \\
Non-animal methods for skin sensitization & Mehling et al. (2012) \\
\hline
\end{tabular}

H. M. Bolt $(\square)$

Leibniz Research Centre for Working Environment and Human

Factors at the Technical University of Dortmund (IfADo), 44139 Dortmund, Germany

e-mail: bolt@ifado.de

\section{References}

Burns KA, Korach KS (2012) Estrogen receptors and human disease: an update. Arch Toxicol 86(10):1491-1504. doi:10.1007/ s00204-012-0868-5

Carvalho M, Carmo H, Costa VM, Capela JP, Pontes H, Remião F, Carvalho F, de Bastos ML (2012) Toxicity of amphetamines: an update. Arch Toxicol 86(8):1167-1231. doi:10.1007/ s00204-012-0815-5

Chasapis CT, Loutsidou AC, Spiliopoulou CA, Stefanidou ME (2012) Zinc and human health: an update. Arch Toxicol 86(4):521-534. doi:10.1007/s00204-011-0775-1

dos Santos NA, Carvalho Rodrigues MA, Martins NM, dos Santos AC (2012) Cisplatin-induced nephrotoxicity and targets of nephroprotection: an update. Arch Toxicol 86(8):1233-1250. doi:10.1007/s00204-012-0821-7

Gulcin I (2012) Antioxidant activity of food constituents: an overview. Arch Toxicol 86(3):345-391. doi:10.1007/s00204-011-0774-2

Kim JE, Shin JY, Cho MH (2012) Magnetic nanoparticles: an update of application for drug delivery and possible toxic effects. Arch Toxicol 86(5):685-700. doi:10.1007/s00204-011-0773-3

Landsiedel R, Fabian E, Ma-Hock L, van Ravenzwaay B, Wohlleben W, Wiench K, Oesch F (2012) Toxico-/biokinetics of nanomaterials. Arch Toxicol 86(7):1021-1060. doi:10.1007/s00204-0120858-7. Erratum in: Arch Toxicol. 2012 Jul;86(7):1061

Matés JM, Segura JA, Alonso FJ, Márquez J (2012) Oxidative stress in apoptosis and cancer: an update. Arch Toxicol 86(11):16491665. doi:10.1007/s00204-012-0906-3

Mehling A, Eriksson T, Eltze T, Kolle S, Ramirez T, Teubner W, van Ravenzwaay B, Landsiedel R (2012) Non-animal test methods for predicting skin sensitization potentials. Arch Toxicol 86(8):1273-1295. doi:10.1007/s00204-012-0867-6

Soderlund DM (2012) Molecular mechanisms of pyrethroid insecticide neurotoxicity: recent advances. Arch Toxicol 86(2):165-181. doi:10.1007/s00204-011-0726-X 